\title{
SPECTRAL AND 3D CULTURAL HERITAGE DOCUMENTATION USING A MODIFIED CAMERA
}

\author{
E. K. Webb ${ }^{1,2}{ }^{*}$, S. Robson ${ }^{3}$, L. MacDonald ${ }^{3}$, D. Garside ${ }^{3}$, R. Evans ${ }^{1}$ \\ ${ }^{1}$ University of Brighton, Brighton, UK - (e.webb2, R.P.Evans)@brighton.ac.uk \\ ${ }^{2}$ Smithsonian's Museum Conservation Institute, Suitland, Maryland, USA \\ ${ }^{3}$ Dept. of Civil, Environmental and Geomatic Engineering, University College London, London, UK - \\ (s.robson, ucfslwm)@ucl.ac.uk, dannygarside@outlook.com
}

Technical Commission II, WG II/8

KEY WORDS: Spectral imaging, image-based 3D reconstruction, cultural heritage, camera characterisation, image quality

\begin{abstract}
:
Spectral and 3D imaging techniques are used for museum imaging and cultural heritage documentation providing complementary information to aid in documenting the condition, informing the care, and increasing our understanding of objects. Specialised devices for spectral and 3D imaging may not be accessible for many heritage institutions, due to cost and complexity, and the modification of a consumer digital camera presents the potential of an accessible scientific tool for 2D and 3D spectral imaging. Consumer digital cameras are optimised for visible light, colour photography, but the underlying sensor is inherently sensitive to near ultraviolet, visible, and near infrared radiation. This research presents the characterisation of a modified camera to investigate the impact of the modification on the spectroradiometric and geometric image quality with the intention of the device being used as a scientific tool for cultural heritage documentation. The characterisation includes the assessment of 2D image quality looking at visual noise, sharpness, and sampling efficiency using the target and software associated with the Federal Agencies Digitization Guidelines Initiative. Results suggest that these modifications give rise to discrepancies in computed surface geometries of the order of $\pm 0.1 \mathrm{~mm}$ for small to medium sized objects used in the study and recorded in the round (maximum dimension $20 \mathrm{~cm}$ ). Measuring the spectral response quantifies the modified camera as a scientific device for more accurate measurements and provides indications of wavelengths that could improve documentation based on sensitivity. The modification of a consumer digital camera provides a less expensive, high-resolution option for 2D and 3D spectral imaging.
\end{abstract}

\section{INTRODUCTION}

Spectral and 3D imaging techniques are used for museum imaging and cultural heritage documentation. These techniques provide complementary information, and previous studies have looked at integration for mapping spectral details in 3D, colour accuracy, and material identification. Beyond these explored applications, selected wavelengths within or beyond visible light can provide an enhanced view of an object or feature and could be used to improve image-based $3 \mathrm{D}$ reconstruction especially for objects that would otherwise be problematic to document. Specialised devices for spectral and 3D imaging may not be accessible for many heritage institutions, due to cost and complexity, and the modification of a consumer digital camera presents the potential of an accessible scientific tool for 2D and 3D spectral imaging. Consumer digital cameras are produced for visible light, colour photography, but employ silicon sensors which are sensitive to near ultraviolet (UV), visible (VIS) and near infrared (NIR) radiation. By modifying consumer digital cameras, these devices can provide capabilities for spectral imaging while retaining the same user-friendly properties and interfaces to a wide range of photographic accessories and software. However, if the consumer digital cameras are produced for visible light, colour photography, does the modification impact the resulting spectroradiometric and geometric image quality and can these devices be used as scientific devices for cultural heritage documentation?

This research investigates the integration of spectral and 3D imaging to improve image-based $3 \mathrm{D}$ reconstruction of small to medium sized heritage objects and the use of a modified consumer camera as an accessible scientific tool for documenting and monitoring collections. This paper will describe the characterisation of the camera, focusing on 2D image quality and spectral response, to understand how its modification impacts the resulting spectral imagery and $3 \mathrm{D}$ reconstruction.

\subsection{Spectral and 3D Imaging}

Spectral imaging techniques are used as standard, non-invasive investigation tools for conservation documentation to record the condition, inform the care, and increase the understanding of objects. Spectral imaging records the interaction of light with materials, and techniques used to document cultural heritage objects include reflected infrared (IR), IR reflectography, UV (reflected and fluorescence), multispectral, and hyperspectral imaging. These techniques can detect changes in composition; show past conservation treatments; visualise faded or obscured details; provide manufacture information; reveal underdrawings; and characterise, differentiate, and identify materials.

\footnotetext{
Corresponding author
} 
Three-dimensional imaging allows digitisation to extend beyond the limitations of two-dimensional object documentation producing virtual and physical 3D models. Techniques include range-based techniques like laser and white light scanning and image-based techniques like photogrammetry. These 3D techniques are used in cultural heritage documentation to monitor dimensional change, virtually reconstruct an object, reduce handling and grant access, create custom mounts or repairs, and produce replicas. Photogrammetry, structure from motion and multi-viewpoint stereo tools in particular, are widely used within cultural heritage documentation as they offer an accessible means of $3 \mathrm{D}$ reconstruction.

Many of the spectral imaging techniques are widely used on objects that could be considered two-dimensional, such as paper and paintings. These techniques provide information that is of interest in three dimensions whether for an object in the round or a flat but still three-dimensional object like paper and paintings. Integrating spectral and 3D imaging allows spectral imaging to extend beyond the limitations of the two-dimensional image by mapping local detail from spectral imaging in three dimensions.

Previous studies have investigated the integration of spectral and $3 \mathrm{D}$ image techniques to combine complementary image data for enhanced understanding and analysis. These studies have focused on mapping spectral image data to 3D geometry (Simon Chane et al., 2013), improving colour accuracy (Brusco et al., 2006), colour measurement (Sitnik et al., 2011), and identifying materials (Brusco et al., 2006; Granero-Montagud et al., 2013; Liang et al., 2014). Looking beyond these explored applications, selected wavelengths within or beyond visible light can provide an enhanced view of an object or feature, so these methods could be used to improve the image-based $3 \mathrm{D}$ reconstruction of the object especially for objects that would be otherwise problematic.

\subsection{Modified Cameras}

While accurate and precise instruments exist for spectral and 3D imaging, these specialised devices can be out of reach for many heritage institutions, professionals, and collections in terms of cost and required expertise. Heritage professionals are already using consumer digital cameras for $2 \mathrm{D}$ and $3 \mathrm{D}$ documentation. Silicon sensors, at the heart of consumer digital cameras, are inherently sensitive to near UV and near IR radiation but are optimised for visible light, colour photography by incorporating an IR blocking filter and a colour filter array (CFA) on the sensor. The top layers of the sensor include microlenses, the CFA, and photodiodes. Microlenses above each pixel focus the light to increase effective sensitivity, and the CFA introduces an array of red, green, and blue filters such that some pixels become more sensitive to specific wavebands, and this allows the reconstruction of a full colour image through a process called demosaicing. The IR filter blocks IR radiation which would, in normal colour photography, lead to reduced contrast, and impact tone and colour balance of images.

The main types of camera conversions available are single wavelength, full-spectrum, and monochrome conversions (Davies, 2018):

- Single wavelength conversion: This conversion includes the removal of the IR blocking filter and replacing it with a bandpass filter that reduces the sensitivity to a single range of wavelengths in the UV, VIS or IR regions of the sensor's sensitivity. The CFA is still in place and the transmission of the red, green, and blue filters impact the sensitivity of the camera.
- Full-spectrum conversion: This conversion includes the removal of IR blocking filter and then filtering the light reaching the camera sensor through mounting filters on the lens or filtering the light source. By placing an IR blocking filter on the lens, the camera can also take visible light images, so this conversion provides a highly versatile modification and a wide range of filters can be used. Similarly, to the single wavelength conversion, the CFA is still in place and the transmission of filters impacts the overall sensitivity of the camera.

- Monochrome conversion: This conversion includes the removal of about $5 \mu \mathrm{m}$ of the sensor surface layers, including the microlenses and CFA in addition to the IR blocking filter (('MaxMax Cameras') and D. Llewellyn, personal communication, March 11, 2016). MaxMax, one of the few companies performing the monochrome conversion, uses semiconductor fabrication equipment in order to remove $5 \mu \mathrm{m}$ from the sensor. Similarly, to the fullspectrum conversion, the light reaching the camera sensor can be controlled by mounting filters on the lens or filtering the light source.

Modified cameras are being used for digital UV and IR photography in several fields including zoology, medicine, dermatology, forensics, botany, and entomology (Davies, 2018). Modified cameras are also being used for cultural heritage and archaeology applications. The introduction of modified cameras provided an inexpensive, easy to use, portable, and highresolution option for spectral imaging of cultural heritage objects (Falco, 2009). Guides for conservation documentation included modified cameras for reflected UV and NIR imaging of cultural heritage objects with primarily examples of paintings and paper objects (Dyer et al., 2013; Warda et al., 2011). Falco (2009) focused on the use of a modified camera for reflected IR imaging with examples of revealing underdrawings in paintings and distinguishing materials in a suit of armour. Verhoeven (2008) provided examples of the use of an IR modified camera for aerial archaeological imaging, ceramic sherds, and obscured writing and Verhoeven et al. (2009) presented its use as a flexible and low-cost approach for aerial archaeological reconnaissance. Webb (2017) used a modified camera for reflected IR imaging of three-dimensional objects investigating the potential integration of IR and 3D imaging for object documentation. In addition to reflected IR imaging, modified cameras are being used for visible-induced IR luminescence imaging to detect Egyptian blue (Kakoulli et al., 2017; Verri, 2009) and visible-induced visible luminescence to detect madder lake (Kakoulli et al., 2017).

Falco (2009) and Verhoeven et al. (2009) presented cultural heritage and archaeological applications for modified digital cameras and their studies included characterisation and assessment of the modified cameras. In both cases the IR blocking filter had been removed and replaced with a filter that blocks visible light and passes IR radiation and the CFA had been left in place. Falco (2009) characterised a modified camera by investigating the sensitivity of the device through estimating the relative and total transmission of the CFA, discussing resolution by visually assessing the camera's ability to resolve small features, and discussing contrast and noise. Many of the comparisons and estimations provided rely on an unmodified camera of a different model. Verhoeven et al (2009) measured the spectral response of the modified camera and used this measurement to take advantage of the unequal spectral transmission in the NIR of the red, green, and blue filters and calculate new spectral bands for aerial archaeological reconnaissance. 
This paper will present the characterisation of a modified consumer digital camera with the IR blocking filter and CFA removed by measuring the spectral response and assessing the image quality and performance. The characterisation investigates the impact of the modification on image quality and its use as a scientific instrument.

\section{MATERIALS AND METHOD}

\subsection{Cameras}

The modified camera used for this research is a Canon 5D Mark II. This camera has a full-frame CMOS sensor $(36 \times 24 \mathrm{~mm})$ with a maximum resolution of $21.1 \mathrm{MP}(5,616 \times 3,744$ pixels $)$ and a pixel pitch of $6.4 \mu \mathrm{m}$. The camera has undergone a monochrome conversion including the removal of the IR blocking filter and anti-aliasing filter stack, the sensor cover glass, and about $5 \mu \mathrm{m}$ of the microlenses and CFA, which was provided by MaxMax LDP LLC. To assess the results of the modified camera characterisation an unmodified camera of the same make and model was characterised using the same methods. A Coastal Optics $60 \mathrm{~mm}$ UV-VIS-IR apochromatic macro lens was used with both the unmodified and modified cameras. This is a high performing lens used for forensics, science, and fine art imaging with low aberration and distortion, and no focus shift from UV through IR.

\subsection{Image Quality}

The assessment of the 2D image quality and performance used the US-based Federal Agencies Digitization Guidelines Initiative (FADGI) Digital Imaging Conformance Evaluation (DICE) which includes image targets and analysis software. These best practices and guidelines are used for collections photography and take into account advances of imaging sciences and cultural heritage documentation (Rieger, 2016) and align with Metamorfoze (van Dormolen, 2012). The target and software were selected as an available option that is already being used in museum imaging to assess standard digitization setups.

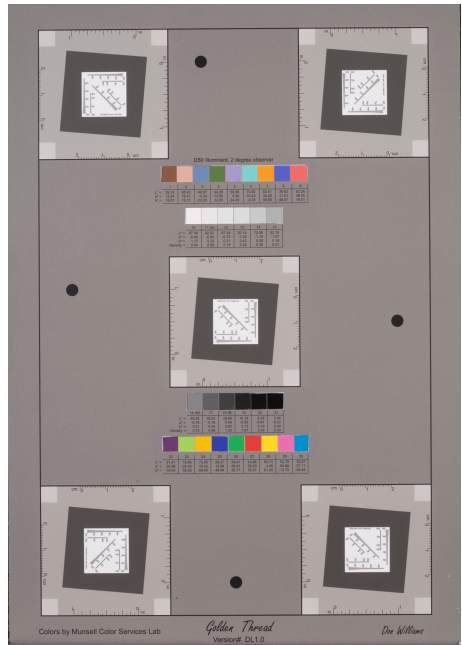

Figure 1. DICE device level target that includes various features to measure image quality and performance of digital imaging systems.

The DICE Device Level Target (Figure 1) made by Image Science Associates was imaged using the modified and unmodified Canon 5D Mark II cameras with a Coastal Optics 60 $\mathrm{mm}$ UV-VIS-IR APO lens, a Canon $50 \mathrm{~mm} \mathrm{f} / 2.5$ compact macro lens, and two Gemini GM400Rx flashes and umbrellas as diffusers. A PECA 916 filter was used to restrict the camera sensitivity of the modified camera to visible light (the filter transmits wavelengths $400-720 \mathrm{~nm}$ ) to provide a comparable sensitivity to that of the unmodified camera. The images were acquired as RAW (*.CR2 Canon files) and processed following the workflow described in Section 2.4. The images were then analysed using Image Science Associates Golden Thread Software focusing on noise, sharpness, and resolution using measures for visual noise, spectral frequency response, and sampling efficiency.

Noise is unwanted variation in light intensity in an image that can impact the reading of the signal. Noise can be caused throughout the imaging chain and by a range of sources and it can be a significant limitation affecting an imaging system and resulting image quality. The DICE target and software measure visual noise from the grey patches on the DICE target, which is expressed as the standard deviation of the signal in the uniform area of the patch.

Sharpness can be considered both a subjective impression relating to the viewer's ability to detect detail and edge transitions and an objective measure of an imaging system's ability to record edges and reproduce contrast especially at high frequencies. Image sharpness is influenced by contrast, noise, and tone reproduction, and can be measured using spatial frequency response (SFR). The SFR measures the contrast loss of an imaging system as a function of spatial frequency and the method used with the DICE software is based on the slanted-edge features in the target. In calculating the SFR, the region of the slanted edge is first located and then the edge spread function and the line spread function (the derivative of the edge spread function) are calculated. The SFR is then derived from the Fourier transform of the line spread function (ISO 12233:2017). The SFR at the $10 \%$ modulation provides a measure for the limiting resolution of the system, and the SFR at the $50 \%$ modulation provides a threshold as a sharpness indicator (ISO 19264-1:2017). The limiting resolution is the smallest distance between image points that can still be resolved (Burns and Williams, 2008). With both the $10 \%$ SFR and 50\% SFR, the aim is to achieve the highest frequency but to not exceed the Nyquist frequency. The Nyquist frequency is the highest frequency that can be reliably reproduced without aliasing and it is the halfsampling frequency or 0.5 cycles/pixel (Allen and Triantaphillidou, 2011; Burns and Williams, 2008). According to the sampling theorem, frequencies below the Nyquist frequency will be faithfully reproduced, but frequencies above the Nyquist frequency will be aliased (Allen and Triantaphillidou, 2011). SFR results can be reported by plotting the modulation level versus spatial frequency.

In comparing multiple SFR results, a convenient measure can be sampling efficiency (Burns and Williams, 2008). The sampling efficiency provides a single value for the comparison of cameras and is the ratio of the limiting resolution to the Nyquist frequency (Burns and Williams, 2008; ISO 19264-1:2017).

\subsection{Spectral Response}

Knowing the spectral response of a camera is important for several applications including colour processing, colour measurement, colour accuracy, and multispectral imaging. However, the spectral response of consumer digital cameras is not provided by camera manufacturers. There are several methods for measuring and estimating the spectral response of a camera system including two standards for characterisation, one produced by the European Machine Vision Associations (EMVA 
Standard 1288 2012) and one published by ISO (ISO Standard 17321-1:2012). The methods presented by these standards include some measurements that require a controlled lab environment and complex, expensive equipment, like the use of a monochromator, that would mainly be available to specialised labs (Berra et al., 2015; Bongiorno et al., 2013; MacDonald, 2015; Prasad and Brown, 2013). While there are several methods for measuring and estimating spectral response, a filter-based method used by MacDonald (2015) presented an accessible option that could include measurements into the NIR for a modified camera. MacDonald compared four methods of measuring spectral response, and the filter-based method was found to have good correspondence with data acquired using a monochromator (2015).

The spectral characterisation of the unmodified and modified cameras followed a method of filtering the camera lens with narrow bandpass filters as per MacDonald (2015). A set of visible filters $(400-700 \mathrm{~nm})$ and NIR filters $(700-950 \mathrm{~nm})$ (Figure 2) were used to acquire a sequence of images of an Avian Technologies Fluorilon-99W white reflectance standard illuminated with a Gemini GM400Rx flash and softbox. The images were processed using DCRAW (Coffin, 2009), an open source software used to decode RAW images. The mean intensity values were calculated from the images, calibrated for the power transmitted through each filter, and normalised with the reflected illumination measurements of the reflectance standard using an Ocean Optics USB2000+ spectrometer.

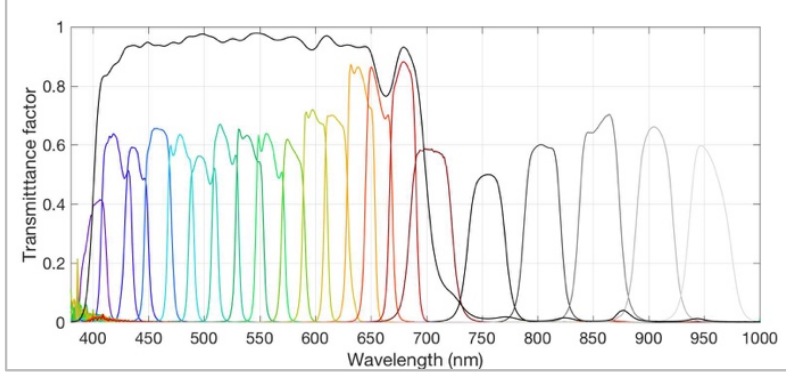

Figure 2. Transmission spectra of VIS and NIR filters used to measure the spectral response of the cameras. The figure includes the transmission of the UV-IR block filter (black), and the figure includes the corrected spectra for the 640,660, 680 $\mathrm{nm}$ (corrected with the transmission of the UV-IR block filter).

The set of visible filters were glass dichroic transmission filters with central wavelengths at intervals of $20 \mathrm{~nm}$ from 400 to 680 $\mathrm{nm}$ inclusive with bandwidths of approximately $20 \mathrm{~nm}$ (Unaxis Optics). The transmission of the visible filters was measured with an Ocean Optics HR2000+ spectrometer (MacDonald, 2015). A visible pass and UV-IR block filter (MidOpt BP550 filter), was used with the 640,660 , and $680 \mathrm{~nm}$ filters to eliminate the effect of sidebands in the NIR. The set of 6 NIR filters (Andover Corporation) are glass filters with central wavelengths at intervals of $50 \mathrm{~nm}$ from 700 to $950 \mathrm{~nm}$ inclusive, with bandwidths of approximately $50 \mathrm{~nm}$. The transmission data for the NIR filters was provided by the manufacturer.

\subsection{RAW Processing Workflow}

A component of the research has been identifying a RAW processing workflow that produces 16-bit linear TIFF files without demosaicing. RAW files are non-standardised, proprietary image formats and require image processing or RAW conversion for a final image file. The RAW conversion generally includes demosaicing, colour space mapping, gamma correction, white balance, noise reduction, anti-aliasing, and sharpening (Allen and Triantaphillidou, 2011; Dyer et al., 2013). Conservation documentation guidelines (Warda et al., 2011) include Adobe Camera RAW (ACR) as an option for RAW conversion and processing; however, if the standard RAW conversion is used to process an image from a modified camera it does not take into account the modification and will process these images as if the image were a 3-channel (red, green, and blue) colour image despite the CFA having been removed. Instead of using ACR, RAW files were processed using DCRAW providing a 16-bit TIFF linear output without demosaicing. The resulting images are scaled using white and black reference points.

While DCRAW and scaling maintain a linear workflow, high magnification viewing of the images revealed a pixel-related pattern, or fixed pattern noise (Figure 3). The pattern could be related to in-camera processing differing between channels or it might be residuals from the removal of the CFA. The pattern is consistent from image to image when a sequence of images is acquired. However, the pattern is not consistent across wavelengths and is most prominent with wavelengths shorter than $550 \mathrm{~nm}$ (Figure 4), hinting that this effect is in fact due to residual spectral selectivity at individual photosites as opposed to processing artefacts. If the images were processed using demosaicing this pattern would be eliminated through averaging, but any increased effective resolution gained from the removal of the CFA would be lost. The source of the pattern has not yet been confirmed, but a flat field correction acquired for each wavelength eliminates this fixed pattern noise and increases the potential effective resolution. The flat field correction has been included in the RAW processing workflow and includes the acquisition of white field images for each filter and illumination source used.

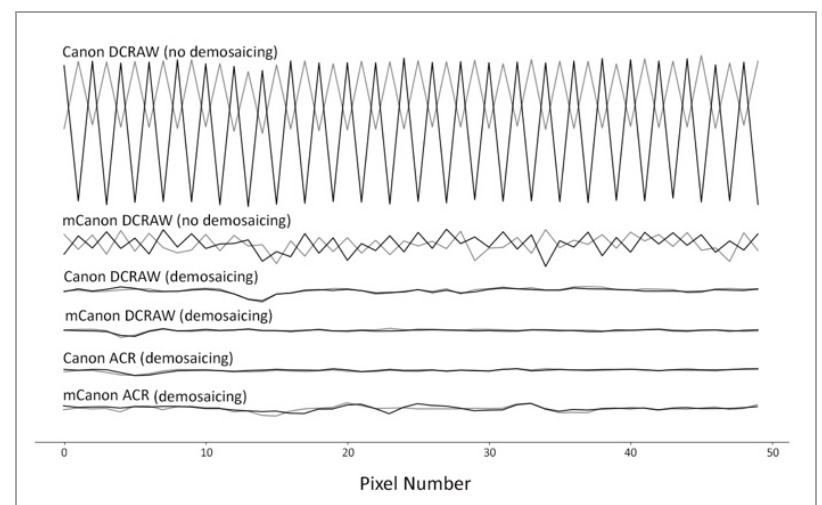

Figure 3. RAW processing and pixel pattern. Vertical profiles of pixel intensities measured from a white patch from unmodified

('Canon') and modified ('mCanon') camera images with different processing with two rows of 50 pixels compared. The top profile shows what a CFA would look like without demosaicing and the second profile shows the fixed pattern noise in the mCanon images processed without demosaicing.

After the flat field correction, the TIFF images are scaled using a black and white point. For the FADGI target the black and white patches are used to map to the $4 \%$ output for the blacks and $97 \%$ output for the whites. The scaling maintains linearity; however, displays are nonlinear so a gamma correction needs to be applied for visualisation. A gamma correction of approximately $1 / 2.2$ was applied providing a resulting image that is visually similar to the results from ACR processing. 


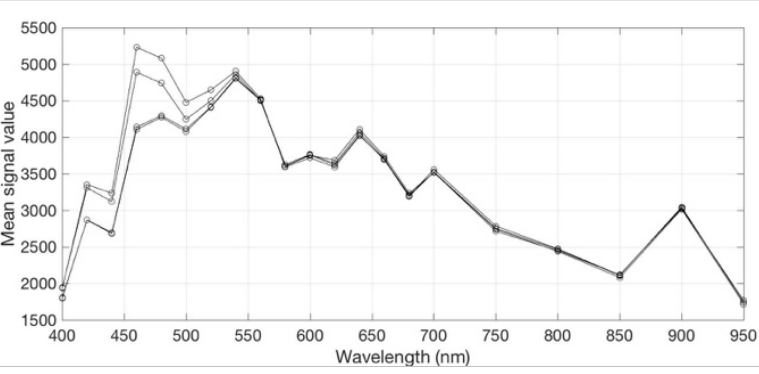

Figure 4. Mean signal value of 100 pixels averaged based on $2 \times 2$ pixel grid. This data has not yet been corrected for the luminous power or the filter sensitivity. The figure shows a difference in spectral response between 400 and $550 \mathrm{~nm}$.

\subsection{D Reconstruction}

Initial tests for assessing the modified camera and resulting 3D reconstruction used a photogrammetric workflow implementing structure from motion and multi-view stereo to document a mango wood vase test object $(19 \times 13 \mathrm{~cm})$ (Figure 5a). The vase included line patterns (graphite and ivory black), pigment patches (Prusssian blue, azurite, malachite, madder lake, zinc white, and titanium white) and varnished areas (shellac and mastic), materials that respond differently to different illumination (UV, VIS, and NIR). The vase was documented using the unmodified and modified Canon 5D Mark II cameras with the $60 \mathrm{~mm}$ Coastal Optics UV-VIS-IR lens. The object was illuminated with two Canon 580 EX II Speedlites with umbrellas to diffuse the light. Three image sets were acquired: visible light images with the unmodified camera (denoted 'VIS'), visible light images with the modified camera using a Peca 916 filter to pass $400-720 \mathrm{~nm}$ (denoted 'mVIS' for modified VIS), and NIR images with the modified camera using a Peca 910 filter (a Kodak Wratten $87 \mathrm{C}$ equivalent) passing wavelengths above $800 \mathrm{~nm}$, (denoted ' $\mathrm{mIR}$ ') (Figure 5b-d).
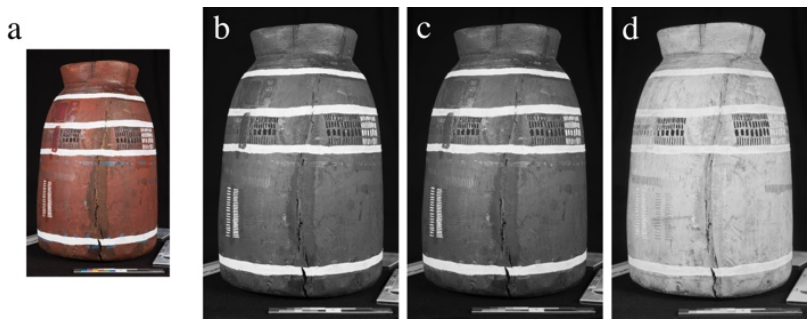

Figure 5. Mango wood vase test object: (a) visible light image from unmodified camera, (b) greyscale visible light image from unmodified camera ('VIS'), (c) visible light image from modified camera and visible pass filter (' $\mathrm{mV}$ is'); and (d) NIR image from modified camera and NIR pass filter ('mIR').

The object was positioned on a manual turntable and images were acquired every $10^{\circ}$ from three camera angles (Figure 6). The turntable allowed for the object to be rotated, while maintaining a constant working distance from camera (mounted on a tripod) to object $(\sim 45 \mathrm{~cm})$ and reducing the handling of the object. Cultural Heritage Imaging (CHI) photogrammetric scale bars were placed around the objects during acquisition for calibration and measurement. Images were acquired as RAW files and converted to grayscale TIFFs using AccuRaw Monochrome. (The RAW workflow included in the following section was not fully developed when this dataset was processed.) The images were used to process the $3 \mathrm{D}$ reconstruction with Agisoft Photoscan Pro and the processing followed an optimised workflow developed in collaboration between $\mathrm{CHI}$ and the US Bureau of Land Management (Schroer et al., 2017) to remove errors and improve the resulting quality. The resulting geometries from visible light and reflected IR imaging were compared using freely available and certified GOM Inspect software.

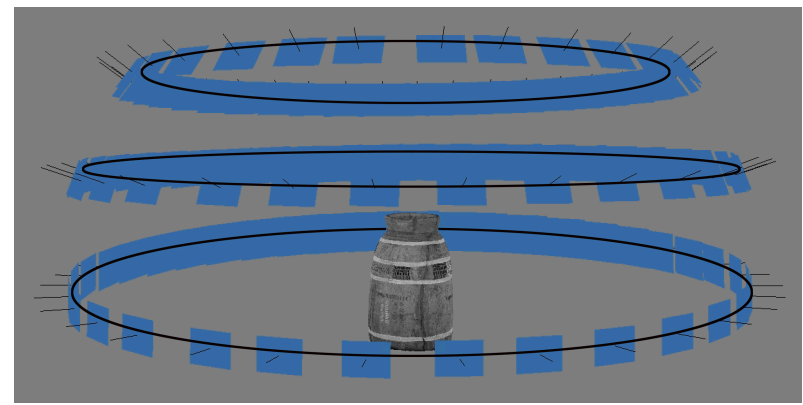

Figure 6. Camera positions for the $3 \mathrm{D}$ reconstruction image sets. The same camera positions were acquired for the three image sets from three camera angles and images every $10^{\circ}$.

\section{RESULTS AND DISCUSSION}

\subsection{D Image Quality}

The image quality assessment investigated the impact of the modification on noise, sharpness, and resolution using measures for visual noise, SFR, and sampling efficiency.

The resulting measures of the visual noise from the DICE target using the unmodified ('Canon') and modified ('mCanon') cameras are presented in Figure 7 with the standard deviation of the digital values of the grey patches plotted against the density of the patches from white (density $=0.04)$ to black (density = 2.42). Three processing options are plotted for both cameras: raw processing using ACR ('ACR'), DCRAW with scaling and gamma correction ('DCRAW'), and DCRAW with flat field correction, scaling, and gamma correction ('DCRAW FF'). The ACR processing results in the lowest levels of noise, consistently falling below a standard deviation of 2 for both the unmodified and modified cameras. Figure 7 shows that the flat field correction for the modified reduces the noise for the lighter patches (density less than 1.67). The noise levels for the modified camera in the dark patches are higher than the unmodified, reaching a standard deviation over 7 in the black patch. Further investigation is needed to investigate this level of noise.

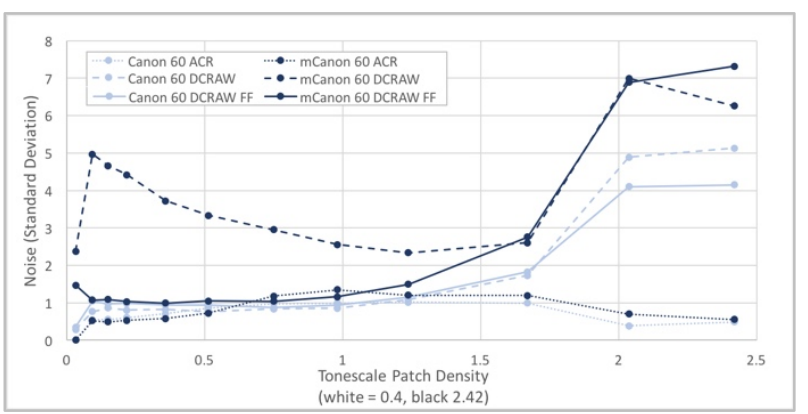

Figure 7. Visual noise measured from the grey patches of the DICE target. The figure plots the standard deviation of the digital values against the density of the tone scale patches from white to black. The figure compares the unmodified ('Canon') and modified ('mCanon') cameras using the Canon $50 \mathrm{~mm}$ ('50') and the Coastal Optics 60mm ('60') lenses and image processing using ACR, DCRAW with scaling and gamma correction ('DCRAW'), and DCRAW with flat field correction, scaling, gamma correction ('DCRAW FF'). 
The DICE target and software offers one option for looking at visual noise from a single image. This is only one type of noise and one way of measuring it, which does not necessarily represent the overall performance of the modified digital camera. Future studies characterising the modified camera should include noise measurement beyond visual noise and single image measures.

The resulting measures of the SFR from the unmodified and modified cameras are presented in Figure 8 as the modulation of contrast plotted against the spatial frequency (cycles per pixel). The plot indicates the limiting resolution at $10 \%$ SFR and the sharpness indicator at $50 \%$ SFR with horizontal lines, and the Nyquist frequency is denoted with a vertical line at 0.5 cycles per pixel. The grey region indicates frequencies above the Nyquist frequency where there is a risk of aliasing.

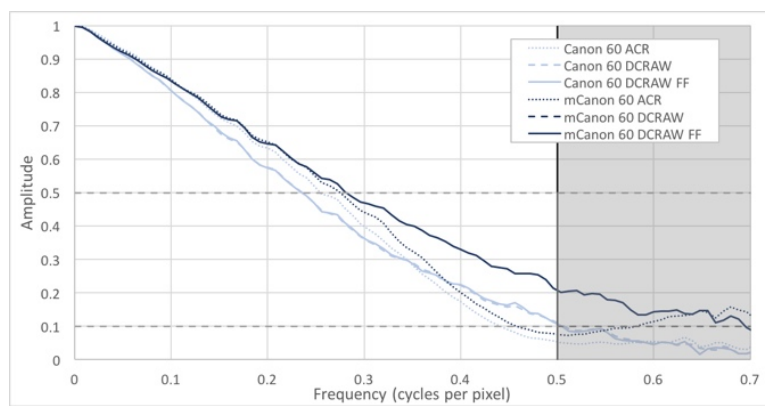

Figure 8. Horizontal SFR curve measured from the slanted edge features at the middle of the DICE target using the unmodified and modified cameras with the Coastal Optics $60 \mathrm{~mm}$ lens. The $10 \%$ and $50 \%$ SFR ranges are marked by dotted horizontal lines and the Nyquist frequency is marked by a vertical black line at 0.5 cycles per pixel. The grey region indicates frequencies that are at risk of aliasing.

The ACR processing for the unmodified and modified cameras using the Coastal Optics 60mm lens ('Canon 60 ACR' and 'mCanon 60 ACR') have the lowest frequencies at $10 \%$ SFR. The DCRAW processing for the unmodified camera with scaling and gamma correction ('Canon 60 DCRAW') and flat field correction ('Canon 60 DCRAW FF') bring the frequencies close to the Nyquist limit, but not above. However, the modified camera with the DCRAW processing with scaling and gamma correction ('mCanon 60 DCRAW') and flat field correction ('mCanon 60 DCRAW FF') push the frequencies over the Nyquist limit.

The results for the sampling efficiency for the unmodified ('Canon') and modified ('mCanon') cameras with the Canon $50 \mathrm{~mm}$ ('50 mm') and Coastal Optics $60 \mathrm{~mm}$ ('60 mm') lenses are presented in Figure 9. The figure compares two processing options, the ACR processing ('ACR') and the DCRAW processing with flat field correction ('DCRAW FF'). The DCRAW processing increases the resulting sampling efficiency as compared to the ACR processing. The Costal Optics $60 \mathrm{~mm}$ lens results in a higher sampling efficiency than the Canon 50 $\mathrm{mm}$ lens for the both unmodified and modified cameras. The modified camera with the Coastal Optics $60 \mathrm{~mm}$ lens results in a sampling efficiency over $100 \%$, which should not be theoretically possible.

The Coastal Optics $60 \mathrm{~mm}$ lens is a high-quality lens with low aberration and distortion, so it can be expected that it performs better than the Canon $50 \mathrm{~mm}$. This is supported with the higher frequencies seen in the SFR curves and the higher sampling efficiency, but does not provide the full explanation for the SFR above the Nyquist frequency and a sampling efficiency over $100 \%$.

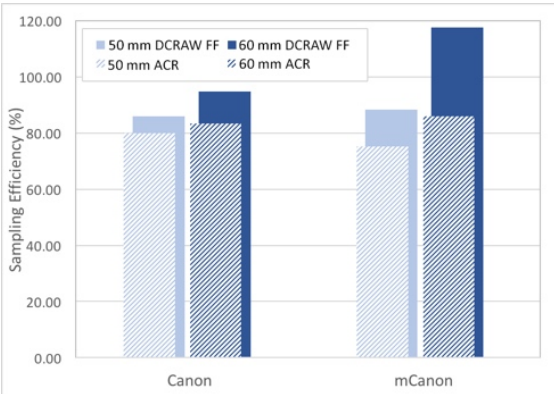

Figure 9. Sampling efficiency (\%) of the unmodified ('Canon') and modified ('mCanon') cameras with the Canon $50 \mathrm{~mm}$ ('50') and the Coastal Optics $60 \mathrm{~mm}$ ('60') lenses. The image processing included ACR ('ACR') and DCRAW with flat field correction, scaling, and gamma correction ('DCRAW FF').

The modification of this camera included the removal of the CFA and the anti-aliasing filter, or the optical low pass filter, which both contribute to the increased SFR and sampling efficiency values greater than $100 \%$. The CFA is used in consumer digital cameras to produce colour images using demosaicing to interpolate the colour information from red, green, and blue pixels. The process of averaging used for demosaicing reduces the effective resolution of a colour image. With the removal of the CFA, the image does not need to be processed using demosaicing algorithms and the effective resolution can be higher without this averaging. By incorporating a flat field correction for the residual pattern discussed in Section 2.4, the final image is able to maintain the higher effective resolution.

The anti-aliasing filter is in place over the sensor of consumer digital cameras to reduce frequencies above the Nyquist frequency and prevent aliasing. Aliasing occurs when high frequencies are reconstructed at a lower frequency and can result in artefacts like moiré patterns. The modified camera resulted in frequencies above the Nyquist frequency and a sampling efficiency over $100 \%$. With frequencies above the Nyquist frequency there is the risk of aliasing.

The SFR and sampling efficiency depend on the image processing and handling of the RAW files in addition to the focus of the lens when the image was acquired. The RAW processing workflow previously described increases the sampling efficiency as compared to ACR processing. Having sharp focus is essential for measuring and comparing SFR and sampling efficiency. The Coastal Optics $60 \mathrm{~mm}$ only has manual focusing capabilities on the Canon cameras, which is a consideration for the reproducibility of the measurements.

\subsection{Spectral Response}

The spectral response measures for the unmodified and modified cameras are presented in Figure 10. The unmodified camera results showed the three channels (red, green, and blue) and a cut off in sensitivity at $700 \mathrm{~nm}$, which is to be expected with the IR cut filter in the camera. There is a tail of the red channel in the blues, and while this may look unexpected it is necessary for detecting purples. The modified camera results showed sensitivity from 400 to $950 \mathrm{~nm}$, with the sensitivity of the device at shorter wavelengths around $400 \mathrm{~nm}$ being over four times the sensitivity of the longer NIR wavelengths around $950 \mathrm{~nm}$. 
Ideally there would be a reference dataset for verifying or comparing the resulting measurements. While there are published datasets available, the results are not consistent and there is a question of the accuracy and reliability of the available spectral characterisation data. Manakov (2016) cited methods and measurements for spectral characterisation and acknowledged that none of the methods listed provide information about the resulting accuracy of the characterisation. Darrodi et al (2015) is one of the few publications discussing the uncertainty and error associated with the measurement of spectral sensitivity. Darrodi et al. provided ground truth data for two cameras (Nikon D5100 and Sigma SDMerill). However, there is a difference between spectral sensitivity between cameras and manufacturers and this ground truth cannot be used as a reference for the Canon 5D Mark II measurements.

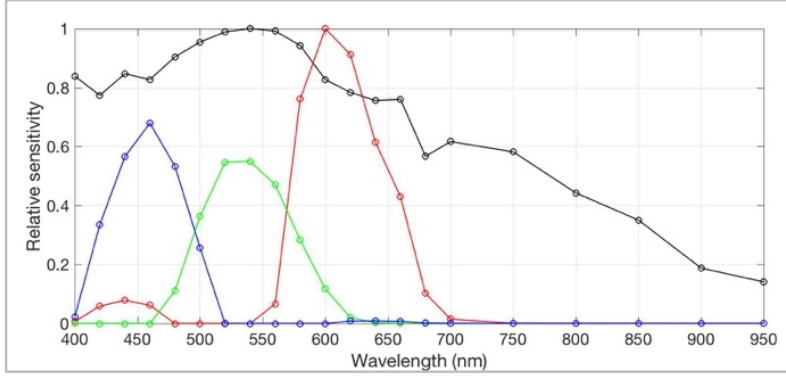

Figure 10. Normalised spectral response for the unmodified and modified camera. The unmodified camera is represented by the blue, green, and red lines for the 3-channels of the camera; and the modified camera is represented by the black line.

The spectral response measurements can help with the understanding of where the camera might perform best with the highest sensitivity. A consideration in using this data is pairing it with the appropriate light source and filters looking at the spectral power distribution of the light source and the transmission measures for potential filters. Areas of lower camera sensitivity, lower output of the light source, and/or lower transmission of the filter can result in image data with increased noise. The spectral response measurements will be paired with future laboratory tests investigating the impact of wavelength on image quality and local image geometry in order to select wavelengths that minimize the surface discrepancy.

\subsection{D Reconstruction}

The three resulting 3D reconstructions of the mango wood vase were aligned and compared using GOM Inspect. Figure 11 includes the surface discrepancy maps for three comparisons of the 3D reconstructions: visible light images from the unmodified camera compared to visible light images from the modified camera with a visible pass filter ('VIS to mVIS'); visible light images from the unmodified camera compared to the NIR images from the modified camera with an IR pass filter ('VIS to mIR'), and visible light images from the modified camera with a visible pass filter compared to NIR images from the modified camera with an IR pass filter (' $m$ VIS to $m I R$ '). The surface discrepancy maps are scaled to discrepancies of $\pm 0.15 \mathrm{~mm}$.

The VIS to mVIS comparison shows good correspondence with a mean discrepancy of $0.03 \mathrm{~mm}$ and a standard deviation distance of $0.15 \mathrm{~mm}$. The largest discrepancies observed in these surface discrepancy maps are the painted white bands and the area around the bottom rim and some of the cracks. In the case of the painted white band, the AccuRaw processing resulted in overexposed areas in lightest regions, and therefore, a loss of geometry in the
$3 \mathrm{D}$ reconstruction. The area around the bottom rim and some of the cracks are where we can see larger discrepancies between the computed models. A contributing factor is that the base of the vase was not recorded.

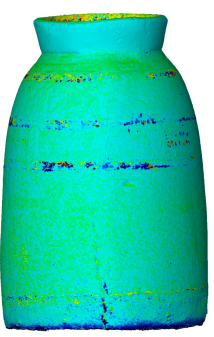

VIS to $m$ VIS

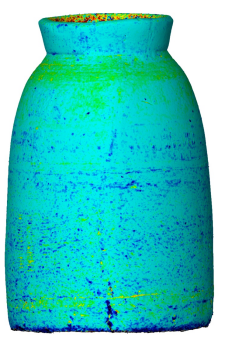

VIS to $\mathrm{mIR}$

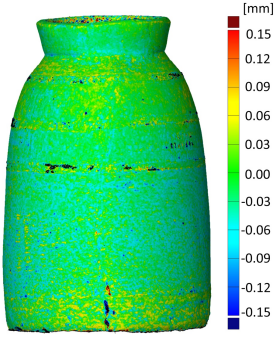

$\mathrm{mVIS}$ to $\mathrm{mIR}$
Figure 11. Surface discrepancy maps comparing the three resulting $3 \mathrm{D}$ reconstructions of the mango wood vase: VIS to $\mathrm{mVIS}$, VIS to $\mathrm{mIR}$, and $\mathrm{mVIS}$ to $\mathrm{mIR}$.

The next stage is to carry out laboratory tests with some well understood reference surfaces in order to fully quantify the outcome of applying these different systems. To establish such references is challenging, not only using objects with well understood geometry but also with a representative suite of surface properties. These will add value to a detailed comparison to AccuRaw and DCRAW workflows for 3D reconstruction.

\section{CONCLUSIONS}

The modification of consumer digital cameras can provide a comparatively cheap and easy-to-use, high-resolution option for $2 \mathrm{D}$ and $3 \mathrm{D}$ spectral imaging, and characterisation of such a camera provides a better understanding of its potential as a scientific imaging tool. This paper described the methods of camera characterisation assessing 2D image quality and measuring spectral response to better understand how the modification impacts the resulting spectral imagery and 3D reconstruction.

Acquiring RAW data is necessary for assessing the 2D image quality, characterising the camera, and using the camera as a scientific device for measurement. The modified camera images without demosaicing resulted in a fixed pattern noise that can be corrected by incorporating a flat field correction. The raw processing workflow utilising DCRAW and a flat field correction requires further work to extend this investigation to characterise the $3 \mathrm{D}$ effect.

The modified camera paired with the high quality Coastal Optics $60 \mathrm{~mm}$ and the raw processing workflow resulted in the frequencies at the limiting resolution (10\% SFR) above the Nyquist limit and a sampling efficiency over $100 \%$. While the raw processing workflow corrected for the fixed pattern noise and allowed for an increased effective spatial resolution, the resulting SFR assessment and sampling efficiencies indicate the risk of aliasing.

The modification increased the spectral sensitivity of the camera as can be expected with the removal of the IR blocking filter and the CFA. The spectral response results showed an increased sensitivity in shorter wavelengths around $400 \mathrm{~nm}$, which is over four times the sensitivity of the camera to the longer NIR wavelengths around 950nm. Measuring the spectral response quantifies the modified camera as a scientific device for more accurate measurements and provides indications of wavelengths that could improve documentation based on sensitivity. 
The outcomes of the characterisation of the modified camera reported in this paper are informing further research on optimising image-based $3 \mathrm{D}$ reconstruction through wavelength selection and depth of field. Whilst demonstrating camera characterisation, the observed 3D discrepancies (max $+/-0.15$ $\mathrm{mm}$ and typically less than $0.05 \mathrm{~mm}$ ) improve our understanding of the capabilities of a modified consumer digital camera as a scientific tool for 3D cultural heritage documentation.

\section{ACKNOWLEDGEMENTS}

This research is part of a $\mathrm{PhD}$ in Heritage Science at the University of Brighton and University College London through the Science and Engineering Arts Heritage and Archaeology (SEAHA) an EPSRC funded Centre for Doctoral Training. Project partners included the Smithsonian's Museum Conservation Institute and Analytik Ltd. Project advisors included Roger Evans, Stuart Robson, Robert Koestler, and Ian Laidlaw with support from Paula DePriest and Lindsay MacDonald.

\section{REFERENCES}

Allen, E., Triantaphillidou, S. (Eds.), 2011. The Manual of Photography, 10th ed. Focal Press, Oxford.

Berra, E., et al., 2015. Estimation of the spectral sensitivity functions of un-modified and modified commercial offthe-shelf digital cameras to enable their use as a multispectral imaging system for UAVs. In: International Conference on Unmanned Aerial Vehicles in Geomatics. ISPRS, Toronto, Canada, pp. 207-214.

Bongiorno, D.L., Bryson, M., Dansereau, D.G., Williams, S.B., 2013. Spectral characterization of COTS RGB cameras using a linear variable edge filter. IS\&T/SPIE ELECT IM, $8660,86600 \mathrm{~N}-86600 \mathrm{~N}-10$.

Brusco, N., et al., 2006. A system for 3D modeling frescoed historical buildings with multispectral texture information. MACH VISION APPL, 17, pp. 373-393.

Burns, P.D., Williams, D., 2008. Sampling efficiency in digital camera performance standards, In: PROC SPIE-IS\&T ELECT IM. pp. 1-5.

Coffin, D., 2009. Decoding raw digital photos in Linux http://www.cybercom.net/ dcoffin/dcraw/ (accessed Aug. 2017).

Darrodi, M., et al., 2015. Reference data set for camera spectral sensitivity estimation. J OPT SOC AM A, 32(3), pp. 381391.

Davies, A., 2018. Digital Ultraviolet and Infrared Photography. Routledge, New York.

Dyer, J., Verri, G., Cupitt, J., 2013. Multispectral Imaging in Reflectance and Photo-induced Luminescence modes: A User Manual.

EMVA Standard 1288: Standard for characterization of Image Sensors and Cameras, 2012. European Machine Vision Association. doi:10.1007/978-3-642-39360-0

Falco, C.M., 2009. High resolution digital camera for infrared reflectography. REV SCI INST, 80.

Granero-Montagud, L., et al., 2013. SYDDARTA: new methodology for digitization of deterioration estimation in paintings. SPIE O3A IV, 8790, pp. 879011-1-9.

ISO 12233:2017 Photography — Electronic still picture imaging - Resolution and spatial frequency responses, 2017. Geneva: ISO.

ISO 17321-1:2012 Graphic technology and photographyColour characterisation of digital still cameras (DSCs) Part 1: Stimuli, metrology and test. Geneva: ISO.
ISO 19264-1:2017 Photography_archiving systems-part 1: best practices for digital image capture of cultural heritage material. Geneva: ISO.

Kakoulli, I., et al., 2017. Application of forensic photography for the detection and mapping of Egyptian blue and madder lake in Hellenistic polychrome terracottas based on their photophysical properties. Dyes and Pigments. 136, pp. 104-115.

Liang, H., et al., 2014. Remote spectral imaging with simultaneous extraction of 3D topography for historical wall paintings. ISPRS J PHOTOGRAMM. 95, pp. 13-22.

MacDonald, L.W., 2015. Determining Camera Spectral Responsivity with Multispectral Transmission Filters, In: COLOR IMAG CONF. IS\&T, pp. 12-17.

Manakov, A., 2016. Evaluation of computational radiometric and spectral sensor calibration techniques, in: $P R O C$ SPIE 9896, Optics, Photonics and Digital Technologies for Imaging Applications IV. SPIE, Brussels.

MaxMax Cameras. https://www.maxmax.com/maincamerapage (accessed Feb. 2018).

Prasad, D.K., Brown, M.S., 2013. Quick Approximation of Camera' s Spectral Response from Casual Lighting, In: IEEE INTL CONF on Computer Vision. IEEE Xplore, pp. 844-851.

Rieger, T. (Ed.), 2016. Technical Guidelines for Digitizing Cultural Heritage Materials : Creation of Raster Image Files, Federal Agencies Digitization Guidelines Initiative. Rochester, New York

Schroer, C., Mudge, M., Lum, M., 2017. Photogrammetry Training: Practical, Scientific Use of Photogrammetry in Cultural Heritage.

Simon Chane, C., et al., 2013. Registration of 3D and Multispectral Data for the Study of Cultural Heritage Surfaces. Sensors 13, pp. 1004-1020.

Sitnik, R., et al., 2011. 3DMADMAC|SPECTRAL: Hardware and Software Solution for Integrated Digitization of 3D Shape, Multispectral Color and BRDF for Cultural Heritage Documentation. Geoinformatics FCE CTU 6, pp. 330-337.

van Dormolen, H., 2012. Metamorfoze Preservation Imaging Guidelines. the Hague. https://www.metamorfoze.nl/ sites/metamorfoze.nl/files/publicatie_documenten/Metam orfoze_Preservation_Imaging_Guidelines_1.0.pdf

Verhoeven, G., 2008. Imaging the invisible using modified digital still cameras for straightforward and low-cost archaeological near-infrared photography. J ARCHAEOL SCI. 35, pp. 3087-3100.

Verhoeven, G.J., et al., 2009. Spectral characterization of a digital still camera's NIR modification to enhance archaeological observation. IEEE T GEOSCI REMOTE. 47, pp. 3456-3468.

Verri, G., 2009. The spatially resolved characterisation of Egyptian blue, Han blue and Han purple by photoinduced luminescence digital imaging. ANAL BIOANAL CHEM. 394, pp. 1011-1021.

Warda, J., et al., 2011. The AIC Guide to Digital Photography and Conservation Documentation, 2nd ed. American Institute for Conservation of Historic and Artistic Works, Washington, DC.

Webb, E.K., 2017. Reflected Infrared and 3D Imaging for Object Documentation. J AM INST CONSERV. 56, pp. 1-14. 